\title{
Hybrid of Fuzzy Logic and Random Walker Method for Medical Image Segmentation
}

\author{
Jasdeep Kaur \\ student (Department of Information Security), CEC, Landran, India \\ Email: jasdeepmavii@gmail.com \\ Associate Prof. Manish Mahajan \\ Associate Professor (Department of IT), CEC, Landran, India \\ Email: cec.manish@gmail.com
}

\begin{abstract}
The procedure of partitioning an image into various segments to reform an image into somewhat that is more significant and easier to analyze, defined as image segmentation. In real world applications, noisy images exits and there could be some measurement errors too. These factors affect the quality of segmentation, which is of major concern in medical fields where decisions about patients' treatment are based on information extracted from radiological images. Several algorithms and techniques have developed for image segmentation and have their own advantages and disadvantages. Random walker method is a supervised segmentation method and it requires that it should be more efficient in producing effective segmentation results in case of medical images which are complex images. In the present paper, we are going to incorporate the advantages of fuzzy logic with a random walker to make resulting segmentation better in texture and quality. For this, we will use fuzzy rules to approximate boundaries in images which will improve segmentation results.
\end{abstract}

Index Terms - Image Segmentation, Random Walker for medical images, Fuzzy Logic.

\section{INTRODUCTION}

Images are the most significant vehicle of expressing information. In this computer vision arena, the information got from images can be used for different critical tasks, for instance: robots navigation, extracting lesion tissues from body scans, detection of malign cells, and recognition of important places (for example Airport) from remote sensing data. Above tasks require a method, which help us to understand images and get information to fulfill our requirements. Image segmentation is best for above requirements, hence, is an initial step in the image analysis process. Image segmentation partitions an image into multiple segments (set of pixels) based on some homogeneity criteria (like color, intensity or texture) to locate and identify objects and boundaries in a digital image [1].

\section{A. Segmentation Types}

Practically, image Segmentation helps in filtering digital noisy images, medical tasks (Locate tumors and other pathologies, Quantify tissue volumes, Computer guided surgical treatments, Diagnosis, Treatment plan), Locate places in satellite images (airports, roads, forests etc.), recognition tasks (Face Recognition, Fingerprint Recognition, etc.). In the literature, there are many methods for segmentation. Each segmentation technique has different advantages and disadvantages for distinct images and the selection of a segmentation method is determined by the particular type of image and the problem's features [2].

For segmentation, we use a plethora of segmentation technologies. Based on two attributes of images, these methods are grouped into two categories [1].

1. Detecting Image Discontinuities: In this, an image is segmented when intensities in image changes abruptly, image segmentation algorithm like edge detection fall in this category [3, 4].

2. Detecting Image Similarities: this class of image segmentation divides a digital image into regions which are similar according to pre-specified measures; the example of this type includes image segmentation algorithms like thresholding [4], region growing, region splitting and merging $[5,6]$.

\section{B. Random Walker Method}

The random walker algorithm is a method for image segmentation. It is based on partial differential equation (a supervised segmentation method) which means user input effects segmentation. This input comprises of seeds (labeling a pixel) i.e. the areas where the user defines whether that area belongs to object which one want to segment or not. Now the random walks start from the unlabeled pixels and the probability is computed to belong to the object. If a user marks $\mathrm{K}$ seeds have different label, then it becomes important to calculate the probability for every pixel that a random walk starts from a pixel will first arrive at each seed. This may be determined by solving a system of linear equations. When probabilities got calculated, the pixel is allotted to the label for which it is most potential to send a random walk. The digital image is treated as a weighted graph [7], 
in which every pixel is like a node which is joined to neighboring nodes by edges, and the changes in image intensities are mapped to edge weights to reflect the similarity between the pixels.

The rest of the paper is organized as follows: Section II gives the overview of Fuzzy Logic including Fuzzy Set Theory and Fuzzy System. A detailed description of the proposed method is presented in Section III. In Section IV, experimental results are described. Finally, section V concludes the research work done in this paper and gives future prospects for further research in this area.

\section{FUZZY LOGIC}

Fuzzy logic provides mathematical rules and functions which permitted natural language queries. Fuzzy logic gives ways to calculate intermediate values within absolute truth and absolute false with resulting values between 0.0 and 1.0.It figures the shades of gray within black/white and true/false. It is a substitute to the A-ornot-A, Boolean 1 and 0 logic definitions made by society. Figure1 depicts the basic difference between fuzzy and Boolean logic.

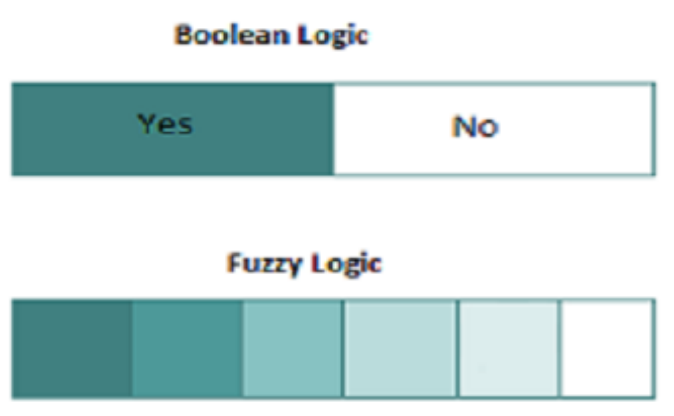

Fig 1. Boolean Logic VS Fuzzy Logic

Information is much ambiguous or inaccurate in the real world. Human thinking filters and translates information in order to arrive at conclusions or to dismiss it as inconclusive. Although machines cannot still deal with imprecise information in the same ways that humans do, in that case fuzzy logic plays an important role as it is basically a type of logic that recognizes more than simple true and false values. Computer programs with fuzzy logic are turning quite useful when the sheer volume of tasks defies human analysis and action. So, a methodical and efficient method for addressing problems of imprecise information is called as fuzzy logic. The data are viewed as fuzzy sets [8] [9].

In various image processing applications, for instance, object recognition and scene analysis, expert knowledge have to be used. Fuzzy set theory and fuzzy logic, offer powerful tools to symbolize and process human facts in shape of fuzzy if-then rules, which also help in image processing tasks. In image processing many problems occur because the data, tasks, and the results are uncertain. That uncertainty, though, is not constant due to the unpredictable, but to the inborn ambiguity and vagueness of image data. Alongside unpredictabilityother kinds of flaw in image processing include grayness ambiguity, geometrical fuzziness, and undefined knowledge of image characteristics.

The nature of these problems is fuzzy. The questions whether a pixel ought to become darker or brighter than it already is, the decision about boundary between two image segments, and indicating whether a tree is there in a picture or not, are instances where fuzzy logics can be used.

\section{A. Fuzzy Set Theory}

Fuzzy logic and fuzzy thinking take place in sets. These are sets whose elements have degrees of membership [10]. The value zero signifies complete nonmembership, value one shows complete membership, and values in between 1 and 0 , are meant to characterize intermediate degrees of membership (partial membership).

This can be clarified with an example in relation to people and "youthfulness". In that case the set U (the universe of discourse) is the set of people. "Young" is a fuzzy subset defined to determine the degree up to which a person ' $p$ ' can be defined young. In the universe of discourse, we have to allot a degree of membership in the fuzzy subset "Young" to every 'person'. The simplest way to do this is with a membership function grounded on the person's age.

$$
\begin{gathered}
\text { Young }(\mathrm{p})=\{1, \text { if } \mathrm{AGE}>=20, \\
30-\mathrm{AGE}(\mathrm{p}) / 10 \text { if } 20<\operatorname{AGE}(\mathrm{p})<=30, \\
0 \quad \text { If } \operatorname{AGE}(\mathrm{p})>30\}
\end{gathered}
$$

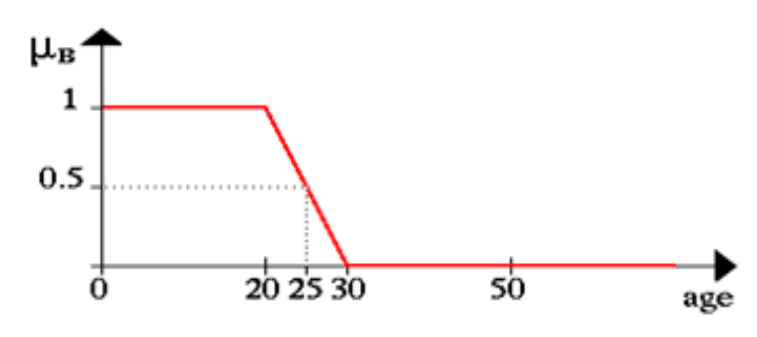

Fig 2. Membership Function

\section{B. Fuzzy System}

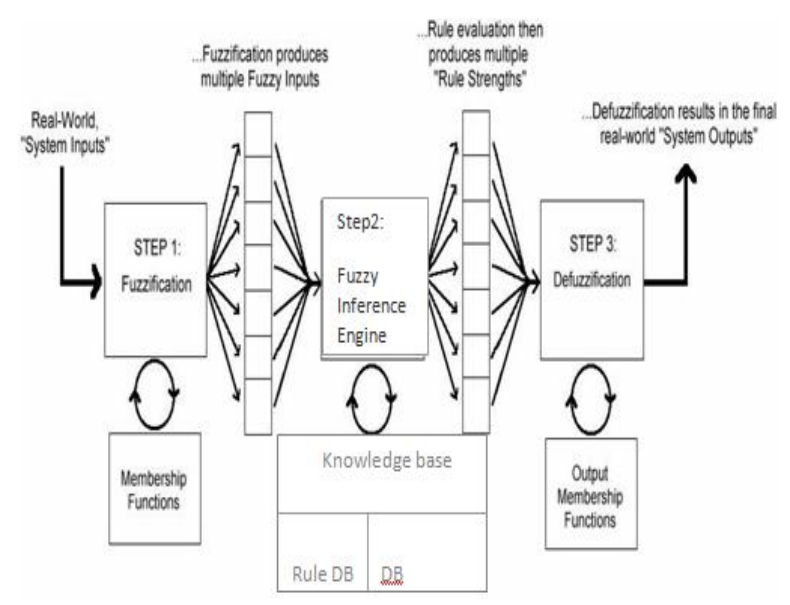

Fig 3. Block Diagram of Fuzzy System 
The above figure depicts a block schematic of fuzzy logic, i.e. basic working of fuzzy system [11] [12]. The steps are described below:

System Inputs: These are crisp values. These are transformed into fuzzy sets in the fuzzification step.

System Outputs: These are the results of defuzzification step, i.e. convert output fuzzy sets back to crisp value.

Fuzzification: In this, crisp values are transformed into the degree of membership for linguistic values, such as, far, near, small, big; of fuzzy sets.

Knowledge base block: This block performs aggregation of propositions having linguistic variables; rules can be specified as:

$$
\text { If }(x \text { is a) and ( } y \text { is } b) \ldots . . \text { then }(\mathrm{z} \text { is } \mathrm{c})
$$

Fuzzy Interface Engine: It combines facts of fuzzification and knowledge base and conduct fuzzy reasoning process.

Defuzzification: This process converts the results back to real world values.

\section{PROPOSED METHOD}

Image segmentation is a topic of higher attention on which there has been a lot of research work is performed. Due to this, thousands of different segmentation methods are there in the literature, still a method is required, which can be efficient in all respects regarding the quality of image segmentation and equally well for different type of images [13]. Similarly, Random walker method [14] has some problems that should be addressed. The results produced by random walker method should need to be more efficient and precise. Also, in this method noise is correlated which makes this random walker method not suitable for many applications as it also segments the noise in the image.

In this paper, to address above mentioned problems we have used fuzzy logic and combine it with the random walker segmentation to make the basic method more effective. The proposed method follows following steps:

Step 1. Input original image which you want to be segmented and generate weights based on image intensities. The segmentation is devised on a weighted graph. In order to represent the image structure (given in the pixels) by random walker biases (i.e., edge weights), one must define a function that maps a change in image intensities to edge weights [15] [16] [17] [18]. In that case we have opted (due to empirical causes) the classic Gaussian weighting function specified with an equation:

$$
v(i j)=\exp \left(-\alpha\left[(h(i)-h(j)]^{2}\right)\right.
$$

Where,

$$
\mathrm{h}(\mathrm{i})=\text { image intensity at pixel } \mathrm{i}
$$

$\mathrm{h}(\mathrm{j})=$ image intensity at pixel $\mathrm{j}$

$\alpha=$ free parameter

Step 2. Obtain a set of pre-labeled (seeds) pixels. These pixels are marked either interactively or automatically. [19]

Step 3. Now random walks start from unseeded pixels and the probability of unlabeled pixels is computed [11].

i. Calculate the probability that a random walker starting at an unseeded pixel $x$ first reaches a seed with label ' $s$ '

ii. Label each pixel with the most feasible seed destination

Step 4. To approximate the boundaries we use fuzzy rules which help us to take a decision about defining boundaries between two segments, which in turn help to produce better segmentation results as compared to earlier methods.

Step 5. After the approximation process weighted graph is build over the boundaries.

Step 6. In the last step we get segmented image finally. It is done by assigning pixel to label for which it has the highest probability.

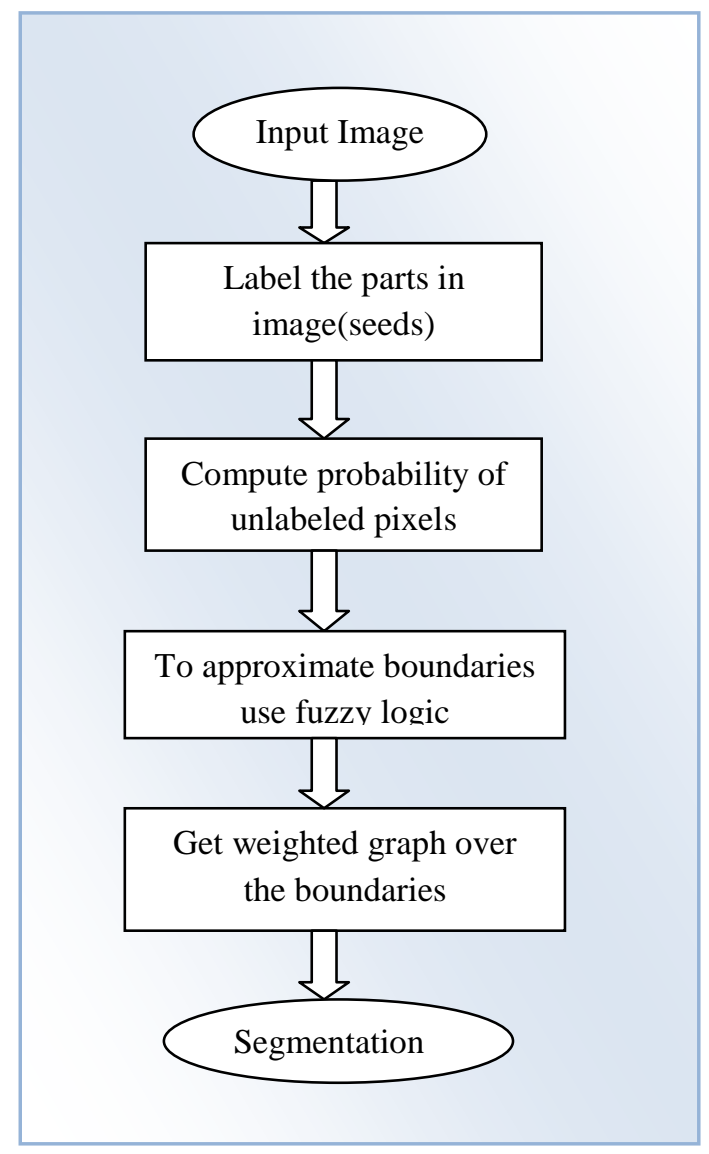

Fig 4. Flow Chart Of Proposed Technique 
In this, we demonstrate the benefits of the proposed method by applying the method to different medical images. The matrices used for the quantitative evaluation of the proposed algorithm were the following [20]:

Bidirectional Consistency Index: It gives a measure that penalizes un-similarity between segmentations in proportion to the degree of overlap.

$$
\mathrm{BCI}=1-\mathrm{BCE}
$$

Where, the Bidirectional Consistency Error (BCE) measure equalizes the segment for every pixel in test segmentation to the minimally overlapping segment containing that pixel in any of the ground-truth segmentations.BCI is defined taking values in $[0,1]$ where value of 1 indicating a perfect match.

Local Consistency Index: It gives the degree of overlap of the cluster related to each pixel in one segmentation and its "closest" approximation in the other segmentation.

$$
\mathrm{LCI}=1-\mathrm{LCE}
$$

Local Consistency Error (LCE) allows for different directions of refinement in different parts of the image. LCI also dwells in the range [0,1] where value of 0 indicates no similarity and a value of 1 indicating a perfect match.

Error Rate (ER): It expresses the ratio of misclassified image pixels over the entire image pixels and is defined as:

$$
\mathrm{ER}=\frac{\mathrm{M}_{\mathrm{f}}+\mathrm{M}_{\mathrm{m}}}{\mathrm{M}_{\mathrm{t}}} \times 100 \%
$$

Where,

$\mathrm{M}_{\mathrm{f}}$ denotes number of false-detection image pixels, $\mathrm{M}_{\mathrm{m}}$ is the number of mis-detection image pixels, and $\mathrm{M}_{\mathrm{t}}$ refer total number of images pixels.
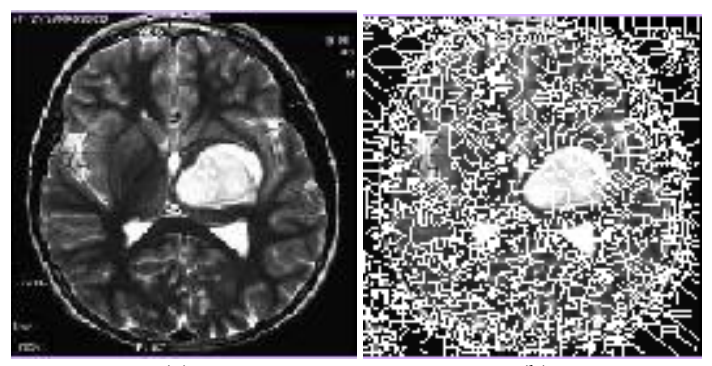

(a) (b)

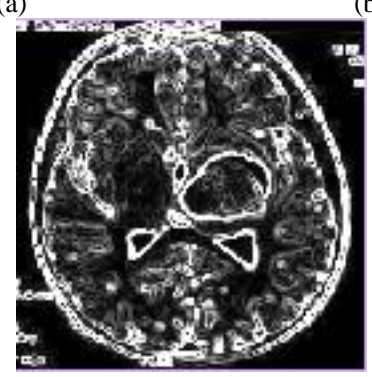

(c)

Fig 5. (a) Original Image (b) Segmented Image using RWM alone (c) Segmented Image using a combination of RWM and Fuzzy Logic

Copyright @ 2015 MECS

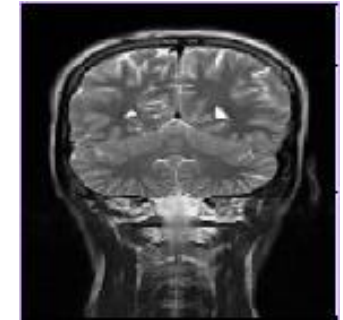

(a)

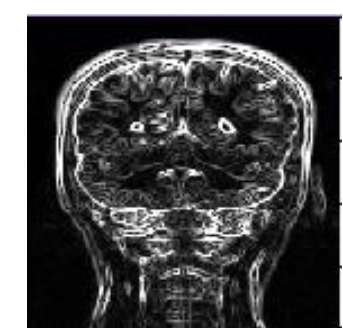

(c)

Fig 6. (a) Original Image (b) Segmented Image using RWM alone (c) Segmented Image using a combination of RWM and Fuzzy Logic

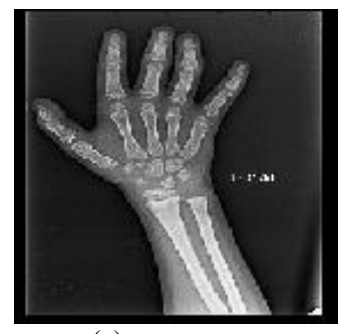

(a)

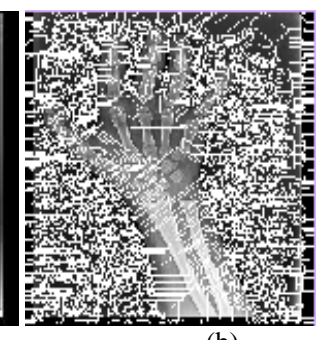

(b)

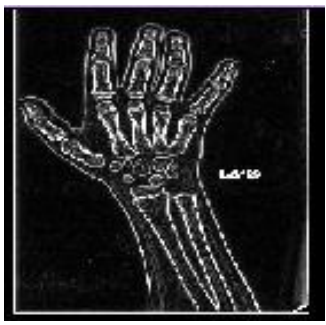

(c)

Fig 7. (a) Original Image (b) Segmented Image using RWM alone (c) Segmented Image using a combination of RWM and Fuzzy Logic

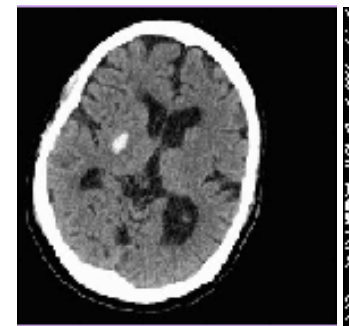

(a)

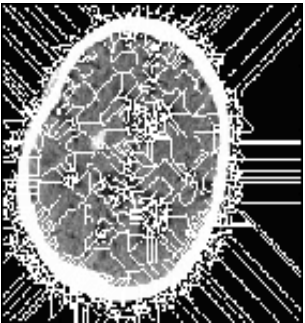

(b)

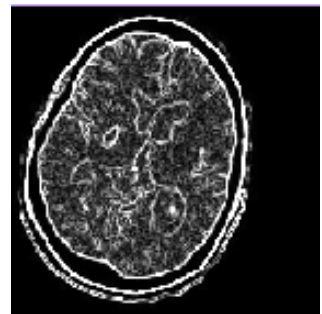

(c)

Fig 8. (a) Original Image (b) Segmented Image using RWM alone (c) Segmented Image using a combination of RWM and Fuzzy Logic 


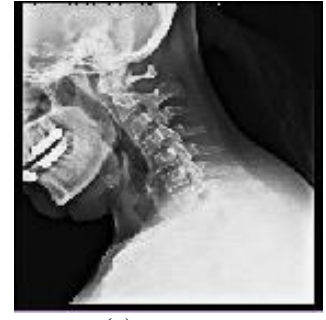

(a)

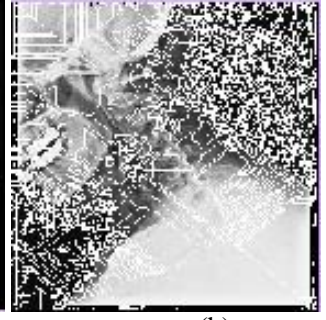

(b)

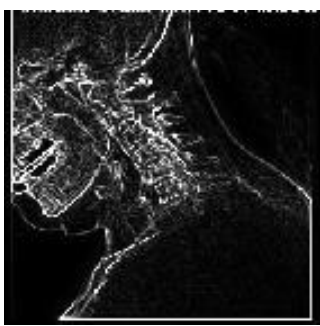

(c)

Fig 9. (a) Original Image (b) Segmented Image using RWM alone (c) Segmented Image using a combination of RWM and Fuzzy Logic

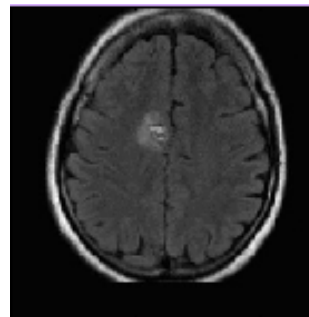

(a)

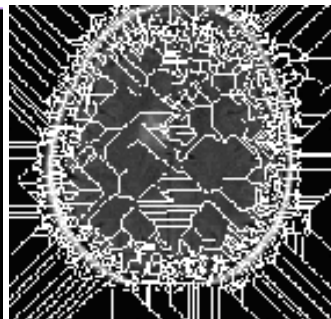

(b)

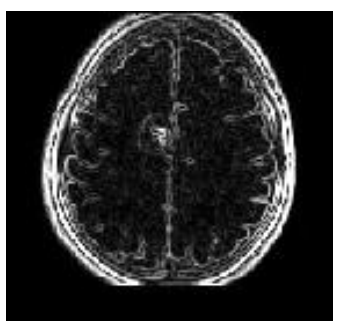

(c)

Fig 10. (a) Original Image (b) Segmented Image using RWM alone (c) Segmented Image using a combination of RWM and Fuzzy Logic

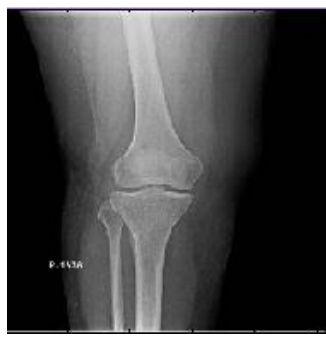

(a)

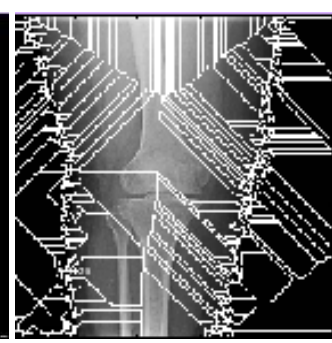

(b)

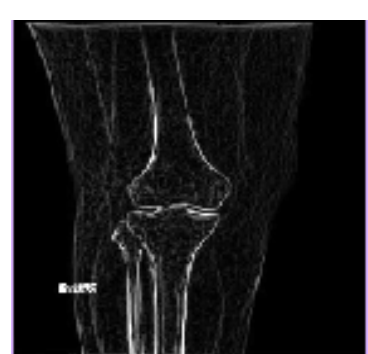

(c)

Fig 11. (a) Original Image (b) Segmented Image using RWM alone (c) Segmented Image using a combination of RWM and Fuzzy Logic

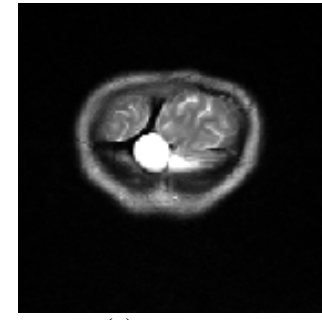

(a)

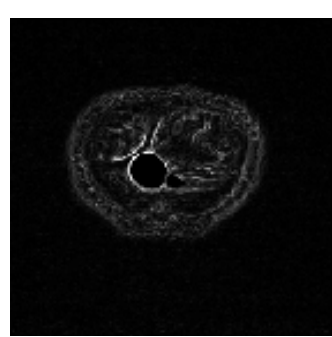

(c)

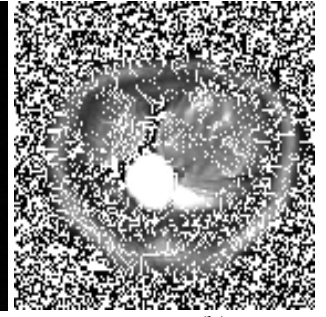

Fig 12. (a) Original Image (b) Segmented Image using RWM alone (c) Segmented Image using a combination of RWM and Fuzzy Logic

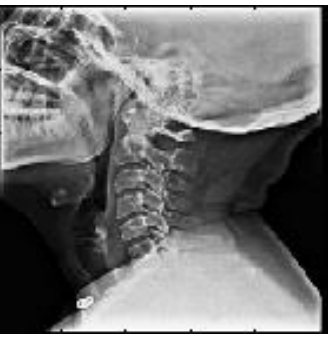

(a)

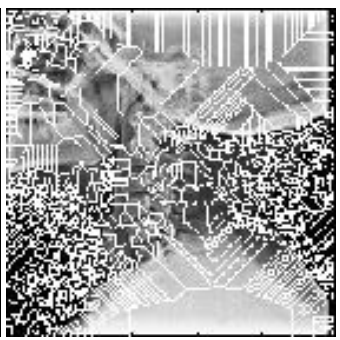

(b)

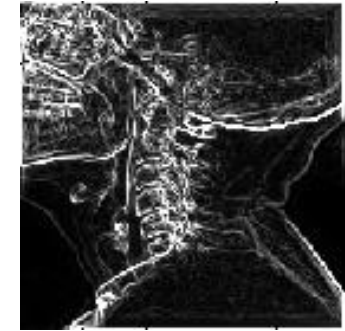

(c)

Fig 13. (a) Original Image (b) Segmented Image using RWM alone (c) Segmented Image using a combination of RWM and Fuzzy Logic

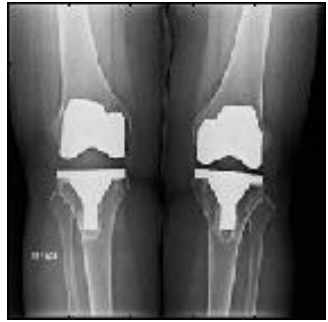

(a)

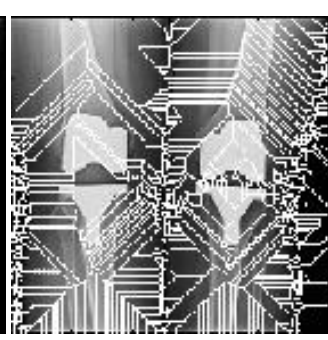

(b)

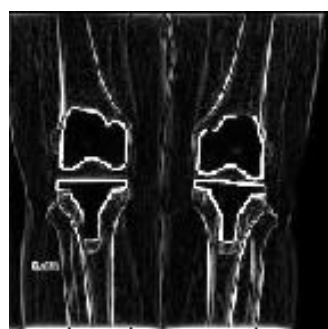

(c)

Fig 14. (a) Original Image (b) Segmented Image using RWM alone (c) Segmented Image using a combination of RWM and Fuzzy Logic

I.J. Image, Graphics and Signal Processing, 2015, 2, 23-29 
Table 1. Comparison of earlier and purposed method based on BCI

\begin{tabular}{|c|c|c|}
\hline Image set & $\begin{array}{c}\text { Proposed } \\
\text { Method }\end{array}$ & Earlier Method \\
\hline Set 1 & 1 & 0.49595 \\
\hline Set 2 & 1 & 0.49605 \\
\hline Set 3 & 1 & 0.49344 \\
\hline Set 4 & 1 & 0.49602 \\
\hline Set 5 & 1 & 0.49326 \\
\hline Set 6 & 0.95286 & 0.45605 \\
\hline Set 7 & 1 & 0.49591 \\
\hline Set 8 & 1 & 0.49156 \\
\hline Set 9 & 1 & 0.49591 \\
\hline Set 10 & 0.87857 & 0.49595 \\
\hline
\end{tabular}

Table 2. Comparison of earlier and purposed method based on LCI

\begin{tabular}{|c|c|c|}
\hline Image set & $\begin{array}{c}\text { Purposed } \\
\text { Method }\end{array}$ & Earlier Method \\
\hline Set 1 & 0.42333 & 0.098833 \\
\hline Set 2 & 0.41667 & 0.098814 \\
\hline Set 3 & 0.73667 & 0.0999326 \\
\hline Set 4 & 0.41833 & 0.098819 \\
\hline Set 5 & 0.77833 & 0.099362 \\
\hline Set 6 & 0.41667 & 0.098814 \\
\hline Set 7 & 0.42667 & 0.098842 \\
\hline Set 8 & 1 & 0.099695 \\
\hline Set 9 & 0.42666 & 0.098842 \\
\hline Set 10 & 0.42668 & 0.098846 \\
\hline
\end{tabular}

Table 3. Comparison of earlier and purposed method based on ER

\begin{tabular}{|c|c|c|}
\hline Image set & $\begin{array}{c}\text { Purposed } \\
\text { Method }\end{array}$ & Earlier Method \\
\hline Set 1 & 2.3622 & 10.1181 \\
\hline Set 2 & 2.4 & 10.12 \\
\hline Set 3 & 1.3575 & 10.0679 \\
\hline Set 4 & 2.3904 & 10.1195 \\
\hline Set 5 & 1.2848 & 10.0642 \\
\hline Set 6 & 2.4 & 10.12 \\
\hline Set 7 & 2.3438 & 10.1172 \\
\hline Set 8 & 0.61287 & 10.0306 \\
\hline Set 9 & 2.3438 & 10.1172 \\
\hline Set 10 & 2.3437 & 10.1171 \\
\hline
\end{tabular}

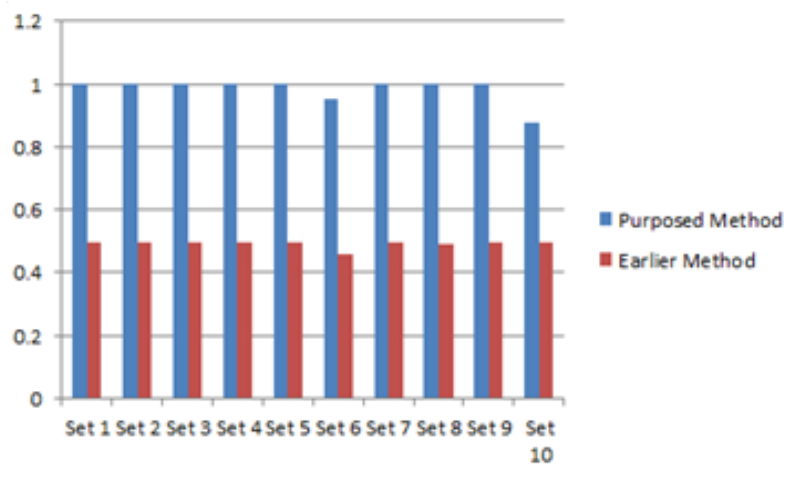

Fig 15. Bar chart comparing values of BCI

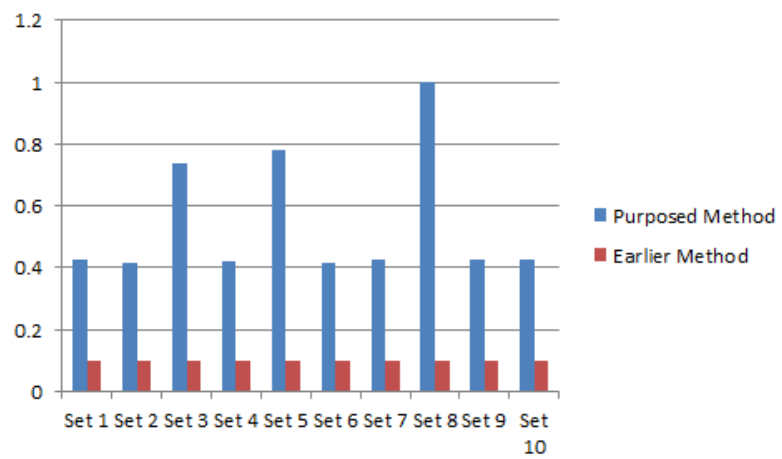

Fig 16. Bar chart comparing values of LCI

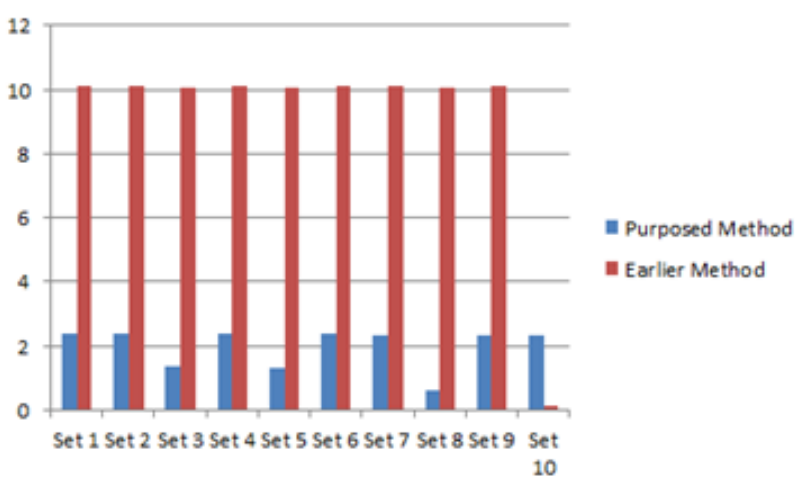

Fig 17. Bar chart comparing values of ER

Above computed values (in tables and bar charts) depicts that BCI and LCI for proposed technique is increased as compared to earlier method which signifies that the texture of resulted image is better after segmentation. ER, has minified i.e. results have lesser number of misclassified pixels, leading to improved image quality.

\section{CONCLUSION AND FUTURE WORK}

Image segmentation has greater use for isolation of images in different segments to diagnose lesions and other important information in an image. In the current paper, we have modified the random walker. A novel random walker algorithm has been presented by combining the features of fuzzy logic with it. We have verified this approach on various medical images and compared this new method with basic random walker method and results found satisfactory. In this new technique, the probability that a pixel belongs to a given seed region is calculated by using fuzzy technique. In future we will apply the fuzzy technique with $\mathrm{K}$ mean clustering and verify the results with this modification.

\section{REFERENCES}

[1] Rafael C. Gonzalez, Richard E. Woods, "Digital Image Processing", 2nd ed., Beijing: Publishing House of Electronics Industry, 2007

[2] K. K. Singh, A. Singh, "A Study of Image Segmentation Algorithms for Different Types of Images", International Journal of Computer Science Issues, Vol. 7, Issue 5, 2010 
[3] N. R. Pal, S. K. Pal, "A Review on Image Segmentation Techniques", Pattern Recognition, Vol. 26, No. 9, pp. 1277-1294, 1993.

[4] W. X. Kang, Q. Q. Yang, R. R. Liang, "The Comparative Research on Image Segmentation Algorithms", IEEE Conference on ETCS, pp. 703-707, 2009.

[5] H. Zhang, J. E. Fritts, S. A. Goldman, "Image Segmentation Evaluation: A Survey of unsupervised methods", computer vision and image understanding, pp. 260-280, 2008.

[6] H. G. Kaganami, Z. Beij, "Region Based Detection versus Edge Detection", IEEE Transactions on Intelligent information hiding and multimedia signal processing, pp.1217-1221, 2009.

[7] P. Doyle, J. L. Snell: Random Walks and Electric Networks, Mathematical Association of America, 1984.

[8] "Neural network, Fuzzy Logic and Genetic Algorithms synthesis and applications", by S. Rajasekaran and G.A. VijaylakshmiPai, (2005), Prentice-Hall, Chapter 7, page 157-221.

[9] "Introduction to Fuzzy Sets and Fuzzy Logic", by M Ganesh, (2008), Prentice-Hall, Chapter 9-10, Page 169233.

[10] "Fuzzy Sets and Fuzzy Logic: Theory and Applications", by George J. Klir and Bo Yuan, (1995), Prentice-Hall, Chapter 12-17, Page169-233.

[11] "Fuzzy Logic: Intelligence, Control, and Information", by John Yen, Reza Langari, (1999), Prentice-Hall, Chapter 5-15, Page 120-603.

[12] "Fuzzy Logic with Engineering Applications", by Timothy Ross, (2004), John Willy and Sons Inc, Chapter 5-15, Page 120-603.

[13] K. K. Singh, A. Singh, "A Study of Image Segmentation Algorithms for Different Types of Images", International Journal of Computer Science Issues, Vol. 7, Issue 5, 2010

[14] TorbenPatz and Tobias Preusser," Segmentation of Stochastic Image with a Stochastic Random Walker Method", IEEE trans. Based on image processing, 21(5), 2012.

[15] J. Shi and J. Malik, "Normalized cuts and image segmentation," IEEEPAMI, vol. 22, no. 8, pp. 888-905, Aug. 2000.

[16] Y. Boykov, O. Veksler, and R. Zabih, "A new algorithm for energy minimization with discontinuities," in Energy Minimization Methods in Computer Vision and Pattern Recognition. Second International Workshop,
EMMCVPR'99, York, UK, 26-29 July 1999. H.-E.-R. Pelillo-M., Ed., 26-29 July 1999, pp. 205-220.

[17] M. J. Black, G. Sapiro, D. H. Marimont, and D. Heeger, "Robust anisotropic diffusion," IEEE Transactions on Image Processing, vol. 7,no. 3, pp. 421-432, March 1998.

[18] X. Zhu, J. Lafferty, and Z. Ghahramani, "Combining active learning and semi-supervised learning using Gaussian fields and harmonic functions" in Proceedings of the ICML 2003 workshop on The Continuum from Labeled to Unlabeled Data in Machine Learning and Data Mining,2003, pp. 58-65.

[19] L.Grady: Random Walks for Image Segmentation, IEEE Trans. on Pattern Analysis and Machine Intelligence, Vol. 28, No. 11, pp. 1768-1783, Nov., 2006.

[20] Hong Ying Yang, Xiang Yang Wang, Qin Yan Wang, Xian Jin Zhang, "LS-SVM based image segmentation using color and texture information," J.Vis.Commun. Image R, 23 1095-1112, 2012.

\section{Authors' Profiles}

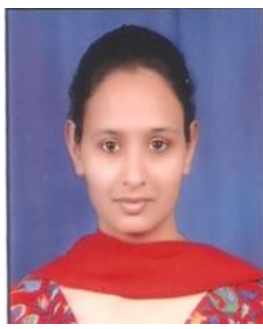

Jasdeep Kaur, received her degree of B.Tech. in the discipline of Computer Science Engineering from Punjab Technical University, Jalandhar, Punjab (India ) in 2012. She is pursuing her M.Tech.(Information Security) from the same university in Chandigarh Engineering College, Landran, Punjab (India ). She has published one review paper and research paper. Her main areas of interest are Digital Image Processing (Digital Image Segmentation) and Information Security

Manish Mahajan, is working as associate professor in Department of IT at Chandigarh Engineering College, Landran, Punjab (India ). He received his degree of B.Tech. from Mullana ( India ) in 2004, and degree of M.Tech. from Punjab Technical University( PTU, Jalandhar ) in 2009. He is currently pursuing his P.hd from PTU. His research interest lies in Digital Image Processing, Data Security and Steganography.

How to cite this paper: Jasdeep Kaur, Manish Mahajan,"Hybrid of Fuzzy Logic and Random Walker Method for Medical Image Segmentation", IJIGSP, vol.7, no.2, pp.23-29, 2015.DOI: 10.5815/ijigsp.2015.02.04 